\title{
NOVASUR, TELEVISIÓN EDUCATIVA DE CHILE
}

(NOVASUR, CHILEAN EDUCATIONAL TELEVISION)

\author{
Blanca Montt Fabres \\ Andrea Villarrubia Martínez \\ Consejo Nacional de Televisión de Chile (Chile)
}

\section{RESUMEN}

En este artículo se da cuenta de Novasur, la televisión educativa de Chile, experiencia de uso de la televisión como recurso pedagógico en el contexto escolar. Novasur pone a disposición de las escuelas un canal de televisión educativa para ser utilizada por los docentes en el aula, en apoyo y estricta relación con el currículo chileno. Esta iniciativa es puesta en marcha el año 2000 por el Consejo Nacional de Televisión de Chile -organismo regulador de la televisión-, como parte de las estrategias por velar por una televisión de calidad. Además de hacer un recorrido explicando el origen, objetivos, servicios y recursos que entrega Novasur, se enfatiza en algunos aspectos que lo diferencian de otras experiencias de televisión educativa en América Latina. En primer lugar, la confluencia de entidades públicas y privadas -como empresas y fundaciones- en la gestión del proyecto. En segundo término, Novasur es mucho más que un canal de televisión, ya que el servicio se plantea desde la convergencia con las nuevas tecnologías a través de su portal web. Como tercer elemento distintivo, Novasur contempla como figura central al docente, tanto por el énfasis puesto en su capacitación -que es un eje fundamental del servicio-, como por el impulso de una retroalimentación permanente entre los profesores y la administración para una construcción conjunta y colaborativa del proyecto. Finalmente, el artículo plantea proyecciones a futuro en un punto de inflexión de este servicio, marcado por la expansión de su operatividad a todo el país.

\begin{abstract}
This article is about Novasur, Chilean Educational Television, an experience of television as a pedagogical resource. Novasur offers an educational TV channel to the schools, to be used by teachers as a support and strictly according to chilean educational programs. This enterprise started in 2000 by the Consejo Nacional de Televisión de Chile -the television regulatory state organization-, as part of its strategies in seeking a quality television. In addition to expose Novasur origins, objectives, services and resources, some unique aspects are highlighted that make it different from other educational television enterprises in Latin America. The first original aspect is the association of both public and private organizations in the execution of this project. Secondly, Novasur is much more than a television channel; as the resource is seen from the convergence with the new technologies, through its website. Third, Novasur puts the teacher in a central role, because of the emphasis put in the training process
\end{abstract}


-one of the central aspects of the project- and the permanent communication and feedback between teachers and the Novasur administration in order to achieve a collaborative development of the project. Finally, the article portrays future projections in a particular moment of expansion of the service within the country.

\section{INTRODUCCIÓN}

Novasur ${ }^{1}$, la televisión educativa de Chile es un proyecto surgido el año 2000 a partir de la iniciativa de organismos públicos y privados con el objetivo de mejorar la calidad y equidad de la educación. A diferencia de otros proyectos televisivos implementados en el continente americano, no depende única o directamente del Estado, ni para su financiamiento, ni para su expansión territorial. Aún cuando es coordinado por el Consejo Nacional de Televisión y los fondos con que cuenta son determinados por la Ley de Presupuesto de la Nación, también recibe aportes de otros organismos como empresas y fundaciones a través de diversos convenios.

Novasur es mucho más que un canal de televisión, es un servicio de televisión educativa. Por ello, la capacitación de los docentes para el uso de esta herramienta pedagógica es una parte fundamental dentro de su concepción, entendiendo que la incorporación de la televisión educativa en el aula necesita mucho más que una buena programación. En este sentido, Novasur intenta dar una respuesta integral a las necesidades didácticas de los docentes, y para eso pone a su disposición programación audiovisual educativa en distintos canales de televisión ${ }^{2}$, un portal web y un sistema de asistencia técnico-pedagógica.

Al iniciar es necesario hacer una distinción. El canal de televisión Novasur proporciona los programas educativos de modo que los centros educativos los graben y formen su videoteca, para utilizarla en función de sus necesidades. Así, el medio que usa el docente en el aula es más bien el vídeo educativo, no la televisión, otorgando flexibilidad a los horarios de clases, la posibilidad de adecuación a los ritmos de estudio de los alumnos y de planificar la clase donde se utilizará este recurso audiovisual.

Siguiendo lo que plantea Cabero (1992), "las posibles virtualidades que puedan tener en el proceso de enseñanza-aprendizaje no dependerán exclusivamente del medio en sí, sino de las relaciones que establezca con otros elementos curriculares: contenidos, métodos, estrategias docentes, contexto de aprendizaje, criterios e instrumentos de evaluación". Se entiende también que "el papel de los medios en el proceso instructivo, no es el de meros recursos o soportes auxiliares, ocasionales, 
Blanca Montt y Andrea Villarrubia

NOVASUR, TELEVISIÓN EDUCATIVA DE CHILE

sino elementos configuradores de una nueva relación, profesor-alumno, aula, medio ambiente, contenidos, objetivos, etc., incidiendo en los procesos cognitivos y actitudinales de los alumnos y transformando incluso los roles de las mismas instituciones docentes" (Sevillano, 1990, 78).

Novasur se plantea como un modelo de desarrollo y gestión que lo hacen único, basado en la integración tecnológica y en la colaboración con los profesores para generar los modelos de implementación y uso pertinente de la televisión educativa.

En esta ponencia expondremos la forma en que se ha llevado a cabo esta tarea y cuáles son las proyecciones para el futuro, marcado por un punto de inflexión que significa su extensión a todo el país.

\section{El PROYECTO NOVASUR}

\section{Surgimiento, historia, contexto}

En los últimos años, el Ministerio de Educación en Chileha desarrolladoun proceso de Reforma Educativa ${ }^{3}$ reformulando los Objetivos y Contenidos del currículum de los distintos niveles de enseñanza, poniendo el énfasis de su política actual en el mejoramiento de la calidad de la educación. Esto significa llevar "la reforma al aula", dándole una especial relevancia al trabajo docente. Así es como todo lo relacionado con recursos pedagógicos que apoyen el proceso de enseñanza y aprendizaje adquiere gran relevancia, en especial las nuevas tecnologías de información y comunicación, acorde también con el propósito de modernizar la educación chilena.

Si bien la preocupación social por la calidad de la educación en Chile está instalada desde hace varios años, cada vez que se dan a conocer resultados de evaluaciones, nacionales (SIMCE ${ }^{4}$ y PSU${ }^{5}$ ) e internacionales (TIMMS), ésta resurge con nueva fuerza, buscando alternativas para revertir los malos resultados académicos. Otro aspecto preocupante es la distribución de estos resultados; en términos generales los colegios municipalizados (públicos) son los que presentan un peor desempeño, mientras que los colegios particulares pagados muestran resultados mucho mejores, dando cuenta de la dificultad para romper con las desigualdades sociales que todavía existen en Chile.

En un contexto donde los niños presentan un alto consumo de televisión en comparación a otros medios ${ }^{6}$, y dado su conocido potencial como recurso de 
aprendizaje, resulta sorprendente que en Chile no existiese una política orientada a potenciar el uso de este medio de comunicación en el contexto escolar, tal como ocurre en otros países de la región, como México o Brasil con larga data.

Es así como el Consejo Nacional de Televisión (CNTV) ${ }^{7}$, respondiendo a su misión de desarrollar una propuesta que contribuya a mejorar la calidad de la televisión, orientándola hacia fines educativos y culturales, desarrolla desde el año 2000 el proyecto Novasur, financiado por privados, inicialmente a través de la Fundación Andes.

Desde su nacimiento, Novasur reúne a actores del ámbito público - el Consejo Nacional de Televisión y el Ministerio de Educación- y otros del sector privado, como el operador de televisión por cable VTR y la empresa de televisión satelital SKY (hoy Directv); Fundaciones y otras que se han incorporado con el tiempo. El CNTV gestiona el proyecto, en términos de su área de competencia: la red territorial $\mathrm{y}$ los recursos financieros.

En la actualidad, sin embargo, Novasur obtiene la mayoría de sus recursos de la hacienda pública. Así, con un presupuesto anual que alcanza los US\$40.000, ha capacitado a cerca de 1.400 profesores de 907 establecimientos a lo largo del país.

Establecimientos Novasur y Profesores Capacitados, según región en número y porcentaje

\begin{tabular}{|c|c|c|c|c|}
\hline \multirow{2}{*}{ Región } & \multicolumn{2}{|c|}{ Establecimientos } & \multicolumn{2}{c|}{ Profesores } \\
\cline { 2 - 5 } & Número & $\mathbf{\%}$ & Número & $\%$ \\
\hline I & $\mathbf{9}$ & $\mathbf{1 . 0}$ & 8 & $0,61 \%$ \\
\hline II & $\mathbf{8}$ & $\mathbf{0 . 9}$ & 129 & $0,61 \%$ \\
\hline V & $\mathbf{9 9}$ & $\mathbf{1 0 . 9}$ & $3,82 \%$ \\
\hline VI & $\mathbf{1 8 0}$ & $\mathbf{1 9 . 8}$ & 306 & $23,31 \%$ \\
\hline VIII & $\mathbf{1 6 4}$ & $\mathbf{1 8 . 1}$ & 309 & $23,53 \%$ \\
\hline IX & $\mathbf{1 7}$ & $\mathbf{1 . 9}$ & 14 & $1,07 \%$ \\
\hline X & $\mathbf{9 7}$ & $\mathbf{1 0 . 7}$ & 91 & $6,93 \%$ \\
\hline XI & $\mathbf{2 4}$ & $\mathbf{2 7 . 3}$ & 377 & $28,71 \%$ \\
\hline Metropolitana & $\mathbf{8 0}$ & $\mathbf{0 . 6}$ & 16 & $1,22 \%$ \\
\hline Total & $\mathbf{9 0 7}$ & $\mathbf{8 . 8}$ & 55 & $4,19 \%$ \\
\hline \hline
\end{tabular}

Tabla 1: Distribución de establecimientos y profesores capacitados por región. Fuente: www.novasur.cl 
Blanca Montt y Andrea Villarrubia

NOVASUR, TELEVISIÓN EDUCATIVA DE CHILE

\section{Finalidad de Novasur}

El Proyecto Novasur tiene como misión el "Contribuir a la promoción social, cultural y educacional de la población chilena, con especial énfasis en el mejoramiento de la calidad y equidad de la educación chilena, a través de un uso convergente de Televisión e Internet en el sistema escolar".

Para avanzar en la misión que Novasur se ha planteado, se definen los siguientes objetivos:

- Poner a disposición de docentes y alumnos de enseñanza básica y media, material audiovisual educativo de alta calidad técnica y pedagógica, destinado a apoyar el proceso de enseñanza aprendizaje en diversas áreas curriculares, tales como: ciencias, lenguaje, humanidades y arte.

- Favorecer el uso convergente de las nuevas tecnologías de información y comunicación en los procesos pedagógicos.

- Apoyar el uso eficiente y pertinente de la programación Novasur en los procesos de enseñanza aprendizaje, de acuerdo a realidades particulares de cada región, comunas y establecimientos.

\section{Modelo de gestión}

Como se mencionó anteriormente Novasur es gestionado desde el Consejo Nacional de Televisión que es el responsable de brindar los recursos necesarios para la dirección operativa. Aporta el director responsable del proyecto y un equipo profesional que cumple con las tareas de coordinación en la preparación de los materiales educativos, la asistencia técnica-pedagógica de la implementación y el desarrollo del portal educativo.

De este modo se puede resumir los participantes de la siguiente manera: 
Blanca Montt y ANDrea Villarrubia

NOVASUR, TELEVISIÓN EDUCATIVA DE CHILE

\begin{tabular}{|c|c|c|c|}
\hline \multirow{3}{*}{$\begin{array}{l}\text { Sector } \\
\text { Público }\end{array}$} & & $\begin{array}{l}\text { Consejo Nacional De } \\
\text { Televisión }\end{array}$ & $\begin{array}{l}\text { Gestión, financiamiento, recursos } \\
\text { humanos, estudios, derechos de } \\
\text { transmisión. }\end{array}$ \\
\hline & & $\begin{array}{l}\text { Ministerio de } \\
\text { Educación }\end{array}$ & $\begin{array}{l}\text { Aportes económicos, y responsable de } \\
\text { prestar apoyo curricular y pedagógico en } \\
\text { el desarrollo del proyecto y en el proceso } \\
\text { de implementación en regiones. }\end{array}$ \\
\hline & & $\begin{array}{l}\text { Televisión Nacional } \\
\text { de Chile }\end{array}$ & $\begin{array}{l}\text { Emisión satelital de la programación } \\
\text { de Novasur desde Santiago a Miami } \\
\text { y de esa ciudad a los establecimientos } \\
\text { escolares. }\end{array}$ \\
\hline \multirow{7}{*}{$\begin{array}{l}\text { Sector } \\
\text { Privado }\end{array}$} & \multirow{3}{*}{ mpresas } & VTR & $\begin{array}{l}\text { Ofrece conectividad gratuita a los } \\
\text { colegios que están dentro de su área } \\
\text { de cobertura, conectando a las escuelas } \\
\text { y transmitiendo una señal con la } \\
\text { programación y también cooperando } \\
\text { con información de su base de datos } \\
\text { sobre material educativo en video. }\end{array}$ \\
\hline & & $\begin{array}{l}\text { SKY Chile } \\
\text { (Hoy Direct tv) }\end{array}$ & $\begin{array}{l}\text { Proporcionó } 100 \text { antenas de televisión } \\
\text { satelital gratuitas para centros } \\
\text { educativos rurales. Emite programación } \\
\text { a través de una señal específica. }\end{array}$ \\
\hline & & $\begin{array}{l}\text { Otros } \\
\text { Cableoperadores }\end{array}$ & $\begin{array}{l}\text { Proveen conexión a las escuelas en } \\
\text { localidades apartadas o en sectores } \\
\text { donde no existe conectividad de VTR o } \\
\text { satelital. }\end{array}$ \\
\hline & \multirow{4}{*}{ Fundaciones } & Fundación Andes & $\begin{array}{l}\text { Aportó con financiamiento durante } \\
\text { el primer año de funcionamiento del } \\
\text { proyecto. }\end{array}$ \\
\hline & & $\begin{array}{l}\text { Fundación Felipe } \\
\text { Herrera }\end{array}$ & $\begin{array}{l}\text { Administra los desembolsos financieros } \\
\text { y el control de presupuesto, entregando } \\
\text { informes periódicos y dedica recursos } \\
\text { humanos con la finalidad de velar por } \\
\text { la calidad técnica de los materiales } \\
\text { educativos. }\end{array}$ \\
\hline & & $\begin{array}{l}\text { Fondo de las } \\
\text { Naciones Unidas para } \\
\text { la Infancia (UNICEF) }\end{array}$ & $\begin{array}{l}\text { Colabora con la estrategia } \\
\text { comunicacional del proyecto, en } \\
\text { producción de eventos, fondos para la } \\
\text { capacitación de profesores y material } \\
\text { educativo audiovisual. }\end{array}$ \\
\hline & & Fundación Chile & $\begin{array}{l}\text { Asesorías en el ámbito educacional y de } \\
\text { investigación tecnológica }\end{array}$ \\
\hline
\end{tabular}

Tabla 2: Aporte de agentes públicos y privados a Novasur 
Blanca Montt y ANDrea Villarrubia

NOVASUR, TELEVISIÓN EDUCATIVA DE CHILE

Novasur establece relaciones con los distintos niveles del Ministerio de Educación, desde su administración central hasta los Departamentos Municipales de Educación (DAEM). En esta alianza estratégica se busca adaptar los requerimientos curriculares que plantea dicho Ministerio, aportando programación pertinente a las necesidades que éste solicita. También, el proyecto busca la colaboración con otras instancias de televisión educativa. Discovery Channel, una de las redes de canales de televisión cultural más grandes del mundo, ha capacitado a grupos de profesores de Novasur en su Modelo de Uso, aprovechando su espacio diario Discovery en la Escuela.

\section{Servicios y Recursos Educativos que ofrece Novasur}

El proyecto Novasur posee un modelo operativo que se grafica a continuación. Esta es la estructura organizacional que permite entregar los siguientes servicios y recursos a los centros educativos adscritos:

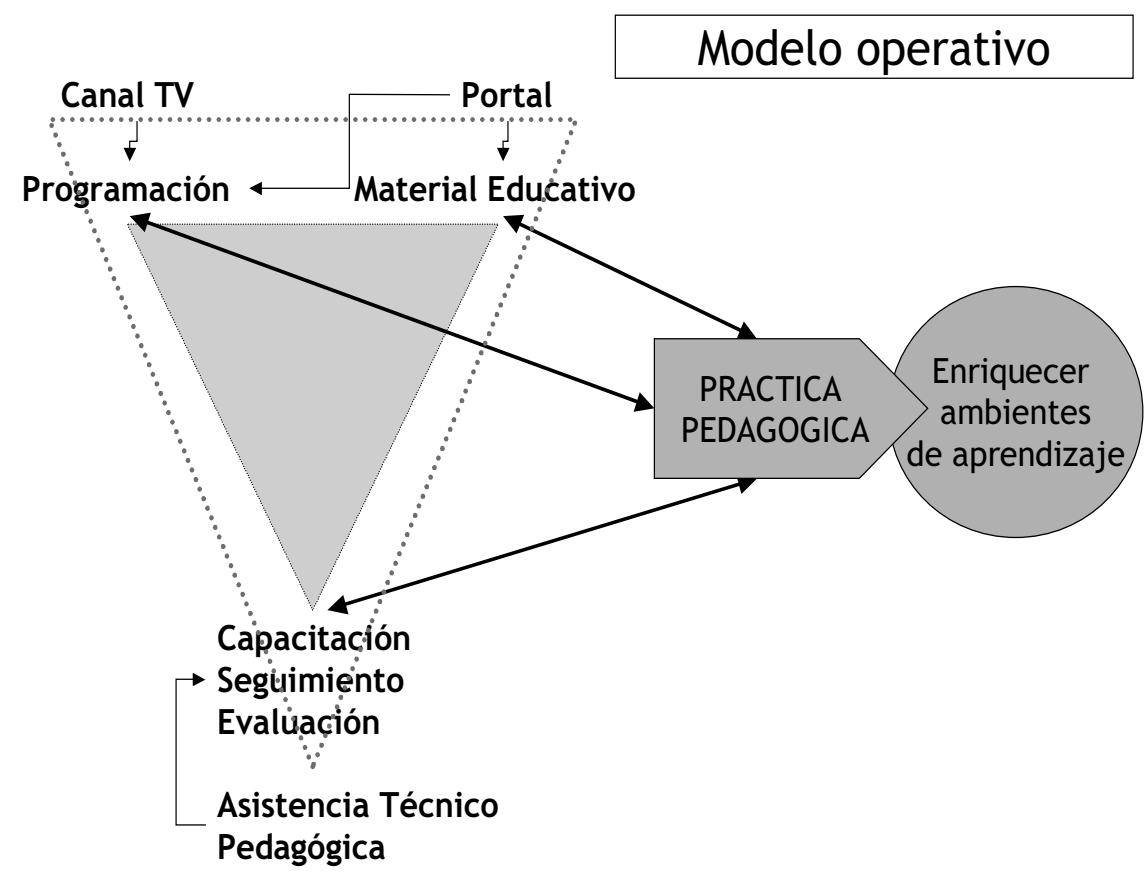

Gráfico 1: Modelo Operativo. Dirección de Novasur. 
De esta manera Novasur puede entregar los siguientes servicios:

1) Canal educativo con programas audiovisuales. La programación de Novasur no se transmite por una frecuencia única, sino a través de los canales que las compañías de televisión por cable y por satélite asociadas al proyecto disponen para la emisión en cada localidad ${ }^{8}$.

La parrilla programática está ajustada al currículo escolar y tiene el formato adecuado para ser usada en la sala de clases. Los contenidos están focalizados en ciencias, lenguaje y comunicación, matemáticas, historia, arte e inglés; también se ha puesto un fuerte énfasis en los objetivos transversales de la educación y el desarrollo de valores, transmitiendo programas útiles para tratar temas de crecimiento y autoafirmación, desarrollo del pensamiento, igualdad de género, respeto a lo pueblos originarios, cuidado del medio ambiente, etc.

Esta programación está dirigida a alumnos de enseñanza básica (primaria) y media (secundaria), se clasifica de la siguiente manera:

- Programas Curriculares. Proponen a los profesores contenidos audiovisuales adaptados a los Objetivos Fundamentales (verticales y transversales) y a los Contenidos Mínimos Obligatorios. Su corta duración (15 minutos aprox.) permite su uso en el tiempo de una clase.

- Programas Complementarios. Tienen un uso más diverso, apoyando el trabajo en otros ámbitos, distintos al estrictamente curricular.

- Franja Abierta. En esta franja se emiten programas orientados a apoyar la educación preescolar, medioambiental, contenidos desarrollados por profesores y espacios vinculados con el Programa de Educación Intercultural Bilingüe, focalizado en el respeto y la identidad de las culturas originarias.

Dentro de la programación curricular, la oferta se ha ido diversificando intentado cubrir todos los subsectores de aprendizaje como se muestra en el gráfico siguiente: 


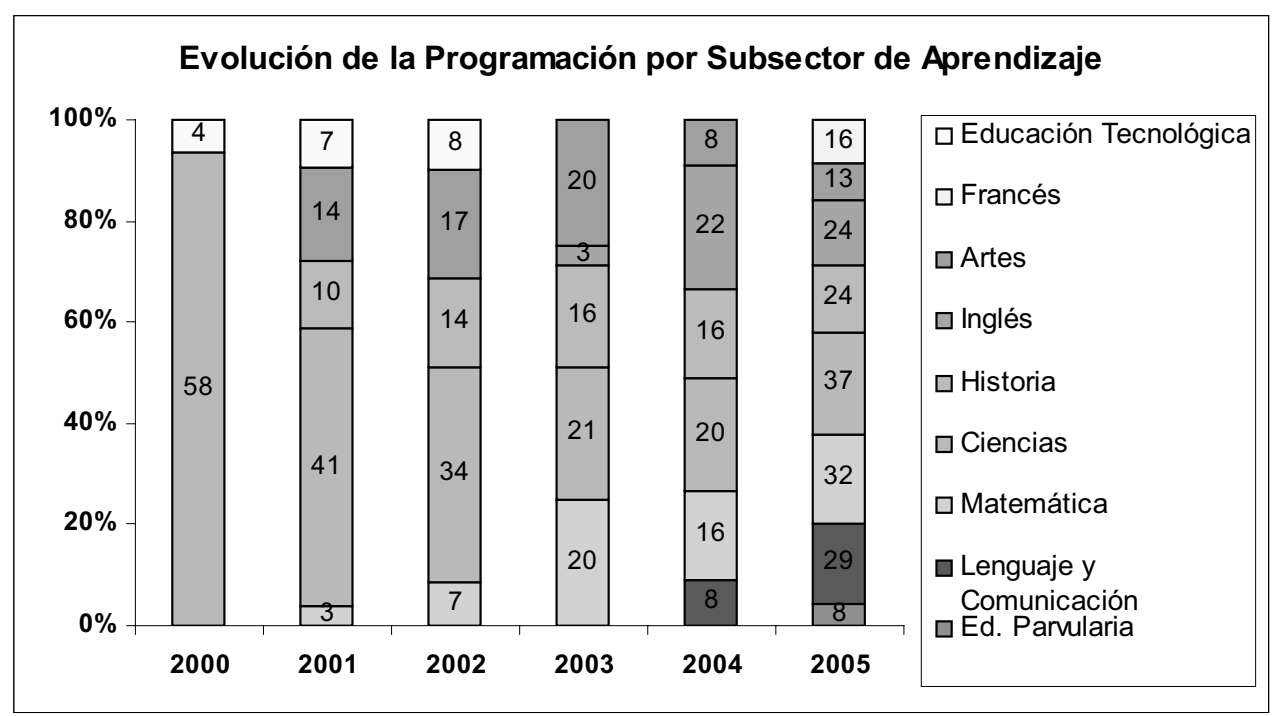

Gráfico 2: Evolución de la Programación por Subsector de Aprendizaje. Área de Programación Novasur

Los proveedores del material audiovisual que se transmite es diverso, la mayoría es de factura extranjera (Channel 4 y BBC de Inglaterra, Transtel de Alemania, TV Ontario y NFB de Canadá, Sesame Workshop-Berlitz de Estados Unidos).

También hay producciones nacionales en su mayoría financiadas por el proyecto y por el Fondo CNTV, que cubre alrededor del 40\% de la programación. Novasur intenta dar respuestas a las necesidades específicas que plantea el Ministerio de Educación. En este sentido, durante el año 2004 Novasur en conjunto con un equipo del MINEDUC desarrolló el espacio "Misión Roflo" una serie de programas destinados a apoyar una Campaña de Lectura, Escritura y Matemáticas (LEM). De esta forma, todas las escuelas básicas pertenecientes al proyecto pudieron acceder a material audiovisual para complementar dicha campaña.

Dentro de la programación se incluyen también notas de facturación propia, de corta duración que buscan capturar y difundir identidades locales, presentando experiencias escolares innovadoras e iniciativas educativas relevantes.

Así es como en total se transmiten 2 horas diarias, de lunes a viernes, lo que al mes permite al establecimiento obtener 10 horas originales y por tanto 80 horas anuales de material audiovisual educativo. 
2) Capacitación y Seguimiento. La capacitación es entendida como un proceso sistemático, secuencial y planificado que implica la realización de un conjunto de transferencias tendientes a habilitar a los profesores para implementar y usar eficientemente este recurso educativo. Complementariamente, la capacitación constituye, también, un sistema de acompañamiento que permite asistir a los colegios en el proceso de implementación del servicio educativo y el uso de los programas educativos en el aula.

Este programa se estructura en tres secciones, la primera describe el proyecto, sus componentes y modalidad de funcionamiento; la segunda da cuenta del modelo de implementación del servicio de televisión educativa; y la tercera sección da a conocer el modelo de uso de los programas educativos en el aula. Este programa tiene las siguientes características generales:

- es semipresencial, por tanto contempla actividades presenciales y a distancia a través de una plataforma e-learning.

- tiene una duración de 120 horas pedagógicas distribuidas en un año, de las cuales 40 horas corresponden a actividades presenciales y 80 horas a actividades a distancia.

La modalidad presencial tiene dos variantes:

- Exposiciones para abordar contenidos de tipo conceptual y metodológico en Jornadas de Capacitación que reúnen regional o provincialmente a los profesores participantes de la capacitación.

- Talleres activo participativos destinados al desarrollo de habilidades prácticas que reúnen comunal o provincialmente a los profesores participantes de la capacitación.

Las operaciones de educación a distancia se implementan a través de una plataforma digital, por medio de una plataforma e-learning de código abierto (Moodle). En esta plataforma se presenta los cursos estructurados en módulos y unidades. Para ello se implementa un sistema de tutoría y de administración de los cursos.

Luego de este proceso de capacitación, es tarea del encargado de Novasur y su equipo de trabajo hacer la transferencia interna, donde la difusión del servicio al interior de la unidad educativa es fundamental. De esta manera, la comunidad educativa debe interiorizarse sobre el proyecto Novasur, sus etapas de instalación, 
Blanca MontT y ANDREa Villarrubia

NOVASUR, TELEVISIÓN EDUCATIVA DE CHILE

componentes, objetivos y justificación. Una vez que se ha difundido Novasur, el profesor encargado tiene la tarea de hacer una segunda transferencia relacionada con el uso de los programas audiovisuales en el aula.

Los profesores encargados de cada escuela en una determinada zona se constituyen en Colectivos Novasur, habitualmente pertenecen a un municipio (o comuna). Los que se reúnen periódicamente para intercambiar experiencias y colaborar en la implementación y el uso del proyecto, y también intercambian material (cintas de vídeo, guías y carteleras).

En algunos casos, por las características de Chile, un país con una geografía accidentada y zonas muy aisladas (sin acceso a Internet o incluso a la electricidad), el Colectivo es una oportunidad fundamental para realizar tareas como la grabación de programas o la obtención del material complementario en la red. En otros casos, los docentes realizan sus propios materiales o utilizan este espacio para elaborar materiales anexos, como por ejemplo tutoriales para el uso de la videoteca.

El Colectivo funciona como una comunidad de aprendizaje ${ }^{9}$ informal, autónoma, colaborativa y con identidad propia. Si bien cuenta con un profesor coordinador, el Colectivo no tiene una estructura definida, una agenda a desarrollar, ni objetivos externos que cumplir. Como comunidades de aprendizaje particulares, cada Colectivo responde a sus necesidades y aborda el proyecto con su propia lógica.

De esta manera, el tipo de apoyo y aprendizajes que se intercambian entre los establecimientos, a través del profesor encargado, dice relación con la implementación y uso de Novasur en los establecimientos educacionales:

- Información. Respecto del funcionamiento y desarrollo de Novasur, de las noticias locales (a nivel comunal o regional) que pudiesen ser de provecho, etc.

- Recursos materiales. Intercambio de cintas para copiar programas que faltan, carteleras, guías pedagógicas, etc.

- Experiencias y percepciones. Respecto de la implementación del recurso en los respectivos establecimientos buscando estrategias exitosas que puedan reproducirse en otros lugares.

- Discursos. En la conversación que se produce en las reuniones de Colectivo se va construyendo un discurso respecto de la televisión educativa, el que se aborda de manera crítica producto de la experiencia reales de los docentes. 
Blanca Montt y ANdrea Villarrubia

NOVASUR, TELEVISIÓN EDUCATIVA DE CHILE

- Apoyo emocional. La labor del docente es una tarea compleja, no exenta de dificultades, por ello el encuentro entre pares representa una verdadera oportunidad de contención.

3) Portal Novasur. www.novasur.cl En la lógica de la convergencia, el portal Novasur sirve de soporte a las necesidades de una comunidad educativa específica, permitiendo a los profesores que forman parte del proyecto relacionarse con el resto de los miembros, a través de un directorio y de los escritorios virtuales para los docentes. En ellos, se pone a disposición de los docentes una herramienta de comunicación personal interna y un espacio de trabajo. Los diferentes foros (nacional, regionales y exclusivo para profesores) recogen la opinión de los docentes en diversos temas de su interés.

Los docentes también pueden acceder a una serie de servicios exclusivos de este soporte, por ejemplo, a los programas educativos en su versión digital, a través de la Videoteca. O al material complementario de Novasur, como las Guías pedagógicas y Carteleras, en el apartado Programación. Aquí, los respectivos buscadores permiten ver el material de meses o años anteriores.

El portal opera como una plataforma que apoya los procesos de capacitación y de seguimiento. En la Biblioteca se encuentran documentos de apoyo a la implementación y uso de Novasur. En el apartado Capacitación. Novasur ofrece a sus docentes cursos a distancia, a través de la Plataforma de e-learning.

Por otra parte, cada región de Chile está representada a través de una página, los Observatorios regionales, que incluye la agenda de trabajo de esa localidad, noticias y una descripción de los colectivos de la región.

\section{Novasur en las escuelas}

Esta propuesta organizacional de Novasur implica una estrategia que gira en torno a los procesos de autorregulación de las comunidades educativas, es decir, el proyecto se implementa siguiendo la dinámica de funcionamiento propia del establecimiento, quien lo adapta a su realidad. Es por ello que la estrategia de Novasur es más bien de oferta, presentación y conversación con la comunidad educativa, luego es ella la que define el modo de implementar el proyecto.

El proceso se inicia con la solicitud que realiza el sostenedor (municipio, corporación o el mismo establecimiento) a Novasur, y de la infraestructura y 
Blanca Montt y Andrea Villarrubia

NOVASUR, TELEVISIÓN EDUCATIVA DE CHILE

equipamiento que posea para mantenerlo. Puede resumir la llegada de Novasur a los establecimientos educacionales de la siguiente manera:

- Los usuarios (municipio, corporación, establecimiento) establecen un convenio con Novasur.

- Los establecimientos son conectados por empresas operadoras de TV cable o satélite.

- Los profesores participantes son capacitados.

- Se establece un sistema de seguimiento del proceso.

- Evaluación de la experiencia.

A su vez, al interior del establecimiento educacional se debe conformar un equipo de trabajo Novasur que permita realizar una serie de tareas que tienen que ver directamente con la implementación del recurso:

- Grabación de los programas

- Almacenamiento de una videoteca

- Administración de la videoteca

- Obtención de las Guías en el sitio web

- Implementación de espacios adecuados para el uso de Novasur en el aula (sala de TV, etc.)

Este equipo Novasur, debe estar coordinado por un profesor encargado, el cual además posee las tareas de divulgar el servicio entre sus colegas, implementar un sistema de uso, participar en las reuniones de su Colectivo, y evaluar periódicamente el material.

\section{Evaluación}

La evaluación de Novasur se aborda desde distintos puntos de vista. A nivel interno hay un dispositivo de evaluación anual, consistente en una encuesta de satisfacción del servicio aplicada a los profesores encargados del proyecto en cada establecimiento, que se contestan en el contexto de Jornadas Regionales. En el caso que no puedan asistir, los profesores tienen la opción de contestar la encuesta a través del portal web. En esta encuesta se indaga sobre la opinión que tienen los profesores respecto de los distintos componentes de Novasur: calidad de la señal, calidad de la programación (programas curriculares y complementarios), calidad de las guías pedagógicas, calidad del portal web y de la asistencia técnica pedagógica ${ }^{10}$. 
Los resultados del año 2005 indican que existe un alto nivel de satisfacción respecto de todos los componentes del servicio. Destacan el material complementario por su alta calificación promedio. A la vez, se aprecia una baja en el promedio del nivel de satisfacción con la asistencia técnica respecto de los años anteriores, que puede explicarse por el cambio de modalidad presencial a modalidad virtual en algunas regiones. El nivel de satisfacción con los programas es alto, la totalidad de los reportes se instalan en los tres niveles superiores (alto, muy alto y óptimo). El material complementario representa la componente mejor evaluada del servicio, todos los reportes se instalan en los niveles más altos de la escala.

A nivel externo también se desarrollan evaluaciones que en su conjunto permiten obtener una visión general del funcionamiento de Novasur: en tanto proyecto, el nivel de implementación en los establecimientos, en el aula, etc. Se presentan aquí en forma sintética las conclusiones de alguno de ellos:

1) Evaluación de la Propuesta Educativa (Universidad de Chile, 2002): Se evalúan los programas audiovisuales y las guías conforman la propuesta educativa de Novasur. Los programas están ajustados al currículo y a través de las guías se transfiere a los docentes el modelo de uso en aula de estos programas. Se compara la percepción de los profesores sobre estos recursos educativos en dos momentos: año 2001 y año 2002. La buena evaluación de la programación y las guías así como su nivel de uso, se han mantenido constantes hasta la fecha, lo que estaría indicando que la propuesta educativa de Novasur es pertinente para los requerimientos de los profesores. Además, las evaluaciones cualitativas indican que para los profesores el uso del material audiovisual por un lado, incrementa la motivación, atención y concentración de los alumnos en los contenidos abordados y por otro, facilita la interactividad y el desarrollo de actividades prácticas en el proceso de enseñanza aprendizaje.

2) Efecto sobre el Aprendizaje de los Alumnos (Pontificia Universidad Católica de Chile, 2000 y 2002): Se constata un efecto positivo de Novasur sobre el aprendizaje de los alumnos. Se observan avances significativos en vocabulario, lectura, estructuración espacial y pensamiento, sin embargo los resultados son menos contundentes que los de la fase piloto del año 2000. Esta diferencia es posible explicarla por la disponibilidad de recursos que había al inicio, lo que permitía un mejor funcionamiento del proyecto en su totalidad, y también con el "efecto novedad" propio de un proyecto que se inicia y que tiende a potenciar los resultados.

3) Implementación del Proyecto en los establecimientos (Universidad Diego Portales, 2003): Estudio realizado sobre una muestra representativa de las regiones 
Blanca Montt y Andrea Villarrubia

NOVASUR, TELEVISIÓN EDUCATIVA DE CHILE

IV, V, VI y X. Los principales resultados indican que: Los programas y las guías son evaluadas con nota 6 , de un máximo de 7 ; El 51,6\% de los profesores consultados expresan un nivel alto de satisfacción con Novasur y el 13,4 \% un nivel muy alto de satisfacción; Según el 59,2\% de los profesores consultados, Novasur le gusta mucho a los estudiantes; El 78\% señala que los alumnos piden que se use Novasur en clases. En orden de importancia los profesores estiman que el uso de contenidos audiovisuales "mejora niveles de conocimiento" (64,1\%), "hacen que estén más atentos en clases" (63,5\%), "permite integrar mejor las materias" $(58,4 \%)$, "aumenta el nivel de retención de los estudiantes" (55,5\%), "estimula la participación” (48\%).

\section{PROPUESTA DE TRABAJO COLABORATIVO}

La propuesta que hoy en día hace Novasur es fruto de un continuo proceso de intercambio de experiencias con los docentes que han incorporado el recurso en sus centros educativos. En los distintos espacios de intercambio (reuniones de Colectivo, Jornadas y Talleres) se ha generado el "know how" de Novasur. Esto posibilita una mayor validez ecológica del proyecto, lo que se traduce en pertinencia y mayores posibilidades de éxito en su implementación. Es así como, en conjunto con los profesores, se desarrollan una serie de trabajos que han permitido construir lo que hoy en día ofrece Novasur.

Como fundamento de este modo de trabajo está la investigación acción, donde la lógica que rige el trabajo con los docentes es un proceso continuo en espiral que pasa por la acción, luego la reflexión, para volver a una acción redefinida. Nos parece una forma de trabajo pertinente ya que, como señala Elliott (1990, p. 24), "la investigación acción en las escuelas analiza las acciones humanas y situaciones sociales experimentadas por los profesores", donde se busca profundizar en la comprensión del profesor de su problema, adoptando una postura exploratoria frente a este.

Como se ha señalado con anterioridad, la incorporación del vídeo educativo dentro del aula es un proceso que implica muchos cambios, pero sobre todo, a nivel de las prácticas pedagógicas. De allí la necesidad de la reflexión sobre la acción ya que, por lo general, las nuevas tecnologías en educación provocan más aversiones que entusiasmos, haciéndose imprescindible que los docentes experimenten en el uso del vídeo en el aula, y a partir de su experiencia definan cuáles son las formas de uso que más se ajustan a las prácticas existentes. Lo mismo al hablar de la incorporación del recurso a nivel de centro educativo en la gestión escolar, es la propia organización la 
que a partir de la experiencia práctica y la reflexión llegará a modelos organizativos ajustados a su realidad.

Presentamos aquí, sintéticamente, las líneas de trabajo que se han desarrollado en conjunto con distintos grupos de los "profesores encargados" de Novasur.

\section{Criterios para una política de Televisión Educativa en Chile}

En la fase piloto de Novasur se llevó a cabo un proceso de investigación acción participativa entre los años 1999 y 2000 con profesores de 200 establecimientos educacionales de enseñanza básica en la VI Región ${ }^{11}$. En esta primera etapa se propone a los profesores una programación audiovisual en ciencias transmitida por televisión (cable y satélite).

En esta investigación-participativa los profesores seleccionados exploran el material recibido, ensayan diversas modalidades de uso, evalúan los programas y las guías de acuerdo a sus propios criterios situacionales. Así, en base a la experiencia y conocimientos desarrollados en el proceso, se establecen y proponen criterios para una política de televisión educativa en sus establecimientos, en sus comunas, en la región y en el país.

\section{Estrategias operativas}

En base a la experiencia de los docentes se han definido algunas estrategias operativas, que desde el punto de vista de los docentes facilitan la implementación de Novasur. Éstas son entendidas más bien como propuestas o sugerencias:

- La incorporación de Novasur en la unidad educativa debe promover una discusión informada sobre los efectos del uso de la televisión educativa. En este sentido, se recomienda aprovechar las instancias existentes o generar espacios que permitan abordar este tema para generar los consensos básicos que le otorguen viabilidad cultural al proceso de incorporación del servicio en el colegio.

- Incorporar Novasur en una escuela exige muchas actividades, por eso se sugiere conformar un equipo de trabajo, que siempre dependerá de las particularidades de cada unidad educativa, como el tamaño, tipo de jornada, etc. Este equipo debe 
Blanca Montt y Andrea Villarrubia

NOVASUR, TELEVISIÓN EDUCATIVA DE CHILE

estar liderado por el "encargado Novasur", quien además es el interlocutor con los coordinadores del proyecto. Además de profesores, se sugiere incorporar a alumnos, codocentes y/o apoderados, debidamente capacitados para asumir las tareas correspondientes: Proponer en la comunidad escolar un espacio de difusión, reflexión y evaluación acerca de la TV educativa; Conformar y administrar una videoteca; Informar regularmente al conjunto de profesores del establecimiento sobre los programas audiovisuales, contenidos y ciclos a los que corresponden; Formarse y formar a otros profesores, alumnos y eventualmente apoderados en modalidades de uso pedagógico de la TV educativa; Gestionar programas de capacitación de profesores, alumnos y apoderados en manejo técnico del equipamiento audiovisual; Coordinación interna y externa.

- Diseñar en conjunto con el equipo directivo, un sistema de funcionamiento interno de Novasur: lugar para la videoteca, un sistema de grabación, sistema de administración, coordinación del salón audiovisual (en caso que sea necesario) y disponibilidad de recursos.

- La incorporación de Novasur debe ser concebida como un proceso que experimenta toda la unidad educativa y no exclusivamente el o los docentes que se encargan de implementar y administrar el sistema. En este sentido se recomienda elaborar una estrategia de difusión que incorpore a todos los estamentos de la comunidad educativa. Con este objeto se recomienda realizar una ronda expositiva inicial que considere espacio para la discusión en las instancias de consejo escolar, equipo directivo, consejo de profesores, reunión informativa con personal administrativo, reunión informativa con personal codocente, reunión informativa con alumnos, y también con los apoderados ${ }^{12}$.

- Una vez que se posea un acopio de material (videoteca bien implementada) desarrollar la transferencia del modelo de uso, dependiendo del tamaño del establecimiento, la infraestructura y los tiempos disponibles, buscar la estrategia que mejor se ajuste a la realidad local. Se sugiere incluir los siguientes contenidos: aspectos técnicos del manejo del televisor y vídeo; sistema de funcionamiento interno; criterios que orientan el uso de material audiovisual (planificación, sistematicidad, convergencia e interactividad).

- La televisión educativa debe formar parte de los proyectos educativos de los establecimientos. $\mathrm{Al}$ incluir Novasur dentro de la política del establecimiento se asegura, por una parte, direccionalidad y consistencia en el uso de esta herramienta, y por otra, su permanencia en el tiempo. 
Blanca MontT y ANdrea Villarrubia

NOVASUR, TELEVISIÓN EDUCATIVA DE CHILE

\section{Modelo de uso de programas audiovisuales Novasur}

La incorporación de los programas educativos de Novasur en los procesos de enseñanza y aprendizaje implica la consideración de algunos elementos, que según sea el caso de cada profesor puede ser un cambio o no dentro de sus prácticas pedagógicas. En conjunto con los profesores que ya tienen experiencia usando el material, se han identificado 4 criterios que son los que orientan el uso de los programas audiovisuales de Novasur:

- Planificación. El recurso es incorporado en los procesos regulares de planificación que se realizan en los establecimientos educacionales, donde se articulan los objetivos fundamentales y contenidos mínimos en relación a un determinado nivel y subsector de aprendizaje. La planificación constituye el carácter más significativo en el uso de programas Novasur y representa un criterio hegemónico puesto que permite regular todos los demás criterios. La planificación del uso de los programas permite otorgar, o al menos intencionar, el carácter sistemático, convergente e interactivo.

- $\quad$ Sistematicidad. Se refiere a la incorporación de esta metodología como una práctica estable, y no como una experiencia aislada y anecdótica, intentando regularizar la exposición de los estudiantes al material audiovisual. Es un uso más bien extensivo que intensivo, en el sentido de que se utilizan de manera dosificada y regular a lo largo del tiempo, ya sea mensual, quincenal o semanalmente.

- Convergencia. Se busca que a partir del uso de programas audiovisuales se intencione el uso de otros recursos pedagógicos, especialmente Internet, enriqueciendo y diversificando así la oferta educativa de los ambientes de aprendizaje.

- Interactividad. Entendida aquí como las interrelaciones que se generan entre los distintos agentes de la comunidad educativa y con los medios (NTIC) a propósito del uso del material audiovisual.

\section{El Portal Novasur}

El portal que hoy en día utiliza Novasur, donde se pone a disposición de los docentes todo el material de apoyo para el uso de los programas educativos, así 
Blanca Montt y Andrea Villarrubia

NOVASUR, TELEVISIÓN EDUCATIVA DE CHILE

como una videoteca con los programas audiovisuales digitalizados, es producto de una investigación conjunta con profesores de la V Región, financiada a través un Proyecto FONDEF ${ }^{13}$.

Este proyecto permitió el desarrollo del portal educativo (uno de los tres componentes que sustenta el servicio que entrega Novasur) y un modelo de uso convergente-interactivo en el aula del material audiovisual digitalizado que se encuentra en dicho portal. También se desarrolló un modelo de guías metodológicas interactivas para apoyar el trabajo de los docentes con los otros medios tecnológicos. Y por último, un modelo de gestión de las capacidades de los centros educativos para incorporar las nuevas tecnologías de información y comunicación en los procesos educativos.

\section{PROYECCIONES A FUTURO}

\section{Expansión de Novasur}

El año 2005 Novasur ha capacitado e incorporado cerca de diez por ciento de las escuelas y liceos del país ${ }^{14}$. Para el 2006 se prevé un amplio crecimiento, completando la expansión en establecimientos de educación primaria y secundaria en la región Metropolitana y en resto del país.

Durante este año es posible que se incorporen a Novasur parte importante de las guarderías públicas, pertenecientes a la Junta Nacional de Jardines Infantiles (JUNJI) y a la Fundación Integra. A su vez, el operador de cable VTR conectará a los que tengan factibilidad técnica a lo largo del territorio.

En términos de programación esta incorporación supone insertar una franja de una hora más, con programas preescolares en consonancia con las prioridades estatales. Esto significa transmitir tres horas diarias. A la vez, por requerimiento ministerial la programación de Novasur enfatizará este año las áreas curriculares de: ciencia, historia, matemática, lenguaje e inglés.

\section{Integración tecnológica de Novasur}

Con la mayor incorporación de establecimientos a lo largo del país, se plantean nuevos desafíos para Novasur en ámbitos como programación, la capacitación, 
el seguimiento y la evaluación. Muchos de estos procesos pueden implementarse a distancia, en la medida en que las escuelas cuenten con tecnología para acceder adecuadamente a la red ${ }^{15}$.

Respecto de la programación, si bien Novasur es un servicio de televisión educativa, deberá plantearse que cada día más escuelas prefieran acceder a la videoteca de programas educativos disponible en línea. Esta situación conlleva además a masificar un modelo de uso distinto de los vídeos, tal como se bosquejó en el proyecto financiado por el FONDEF. También hay que considerar que los soportes digitales presentan ventajas respecto de los analógicos en calidad de imagen y sonido, por lo que con el tiempo es de esperar que las escuelas cuenten con los recursos para incorporar como parte de su equipamiento reproductores DVD y proyectores multimedia.

$\mathrm{Al}$ aumentar el uso de la videoteca digital, sería deseable que los profesores pudieran evaluar los programas al momento de utilizarlos. Para ello se podría disponer de la bitácora de registro de uso en línea, facilitando la evaluación inmediata de los programas utilizados en el aula.

Respecto del seguimiento y la capacitación, en los inicios del proyecto, tanto la capacitación como la coordinación regional de Novasur han sido altamente presenciales en las localidades en las que se ha implementado el servicio. Con el crecimiento de escuelas participantes, la modalidad a distancia adquiere más relevancia y se hace necesario utilizar el portal como una herramienta de trabajo de los docentes. El uso la plataforma de e-learning en el área de capacitación tendría como fines favorecer el autoaprendizaje de los profesores; generar una cultura de la participación y la colaboración entre los docentes - potenciando los foros y otros espacios de discusión-; entregar contenidos relevantes para la implementación del proyecto en las unidades educativas; aumentar la comunicación directa con el equipo profesional de Novasur.

Sin embargo, nos parece importante no perder de vista uno de los aspectos que caracteriza a Novasur: la cercanía con los profesores que se encargan del proyecto en sus respectivos centros educativos. Si bien la relación con ellos, se va virtualizando cada vez más, esto no puede ir en desmedro de un estilo de trabajo colaborativo orientado a la continua mejora del proyecto. Por ello resulta necesario destinar recursos para fortalecer la comunidad virtual, a través de las distintas herramientas que ofrece el portal educativo, así como otras estrategias que pudiesen desarrollarse en esta dirección. 
Blanca MONTT y ANDREa VillarRUbia

NOVASUR, TELEVISIÓN EDUCATIVA DE CHILE

\section{NOTAS}

$1 \quad$ www.novasur.cl

2 Desde el 2006 se transmite a través del Canal del Senado, el Canal de la Cámara de Diputados, y Showiz.

3 La reforma educacional en Chile partió el año 1990, en una primera etapa se construyeron las bases para el mejoramiento del sistema escolar: condiciones laborales de los docentes, condiciones materiales para el aprendizaje (textos, bibliotecas, material didáctico, computadores en red), condiciones institucionales y técnicas para la renovación de la pedagogía y la gestión (Programa P-9oo focalizada a las 900 escuelas con peores resultados, Programas Mejoramiento Enseñanza, etc.). Luego, entre 1996 y 2000 se empezó con la Jornada Escolar Completa y reformas curriculares, junto a una intensificación de algunos programas de mejoramiento (Programa Enlaces, MonteGrande, y fortalecimiento profesión docente). En el 2003 se hace una segunda reforma curricular, dando un hace un giro de las estrategias de desarrollo profesional docente y se pone énfasis en las competencias para la globalización (inglés y TICs). Ver www.mineduc.cl

4 El Sistema de Medición de la Calidad de la Educación (SIMCE) funciona en base a una prueba que se aplica a nivel nacional, una vez al año, a todos los estudiantes del país que cursan un cierto nivel, el cual va alternándose entre $4^{\circ}$ Básico, $8^{\circ}$ Básico y $2^{\circ}$ Medio.

5 Prueba nacional que rinden los jóvenes para postular a las universidades chilenas.

6 Según resultados de la V Encuesta de Televisión realizada por el Consejo Nacional de Televisión (CNTV), los niños menores de 13 años presentan el siguiente consumo de medios: televisión abierta $74.5 \%$; televisión pagada $28.1 \%$; radio $34.2 \%$; Internet 21.7\%; lectura 19\%. Disponible en: http://www.cntv.cl/medios/Publicaciones/2005/ PrincipalesResultadosENTV2005.pdf [consulta 2006, 10 de marzo]

7 Organismo regulador de la televisión en Chile: encargado de entregar concesiones de los canales, resguardar el cumplimiento de la normativa vigente respecto de contenidos de la programación transmitida (incluye la sanción de la violencia, la pornografía y la truculencia), y promover una televisión de calidad para todos los chilenos y chilenas. Ver www.cntv.cl

$8 \quad$ El Cableoperador es VTR y la empresa satelital es Directv.

9 Por comunidad de aprendizaje se entienden aquellas comunidades lo que plantean Pérez, A. y Salinas, J. (2001), “donde el motivo principal de la existencia de dicha comunidad pasa de ser el intercambio y la intercomunicación a ser el aprendizaje y el desarrollo profesional".

10 El instrumento utiliza escalas de 1 a 7 para reportar el nivel de satisfacción de los profesores con los principales componentes. Los resultados del año 2005 indican que existe un alto nivel de satisfacción respecto de todos los componentes del servicio. Destacan el material complementario por su alta calificación promedio y la baja desviación estándar.

11 Con los tres sistemas de dependencia que existen en Chile: municipal, particular subvencionado y particular pagado.

12 Para facilita la descripción y exposición de los elementos centrales del servicio Novasur el profesor encargado recibe un set que incluye documentos, presentaciones en Power Point y videos explicativos. 
Blanca MontT y ANdrea Villarrubia

NOVASUR, TELEVISIÓN EDUCATIVA DE CHILE

13 Fondo de Fomento al Desarrollo Científico y Tecnológico, perteneciente a la Comisión Nacional de Investigación Científica y Tecnológica CONICYT.

14 Según estadísticas del Ministerio de Educación existen 9.206 establecimientos educacionales en Chile, de los cuales 6.736 corresponden a escuelas urbanas y 2.470 a escuelas rurales.

15 Según datos de la Red Enlaces "en la actualidad, aproximadamente el $75 \%$ de la matrícula tiene acceso a Internet, y el 40\% a Banda Ancha. Además durante el 2005 se debe considerar la llegada de Internet al mundo Rural a través del Fondo de Desarrollo de las Telecomunicaciones que permitirá que 600 escuelas rurales cuenten con Banda Ancha”.

\section{REFERENCIAS BIBLIOGRÁFICAS}

Cabero, J. (1992). Análisis, selección y evaluación de medios audiovisuales didácticos. [en línea]. Disponible en: $\quad$ http://edutec.rediris.es/ documentos/1992/qurricul.html [consulta 2006, 10 marzo].

Consejo Nacional de Televisión. (2005). Encuesta Nacional de Televisión 2005 [en línea]. Disponible en http://www. cntv.cl/medios/Publicaciones/2005/ PrincipalesResultadosENTV2005.pdf [consulta 2006, 10 de marzo].

CNTV-NOVASUR. (2000). Criterios para una Política de Televisión Educativa en Chile y para el Uso de Programación Audiovisual en el Aula. Documento interno (inédito). Santiago.

CNTV-NOVASUR. (2003). Criterios que orientan el Uso de Programas Audiovisuales. Documento interno (inédito). Santiago.
Elliot, J. (1990): La investigación-acción en educación. Madrid: Morata.

Pérez, A. y Salinas, J. (2001). Comunidades virtuales: de las listas de discusión a las comunidades de aprendizaje. $V$ Congreso Internacional de Tecnología, Educación y Desarrollo sostenible. 1719 de septiembre, Murcia. Disponible en: http://gte.uib.es/articulo/ CVIRTUALES01.pdf [consulta 2006, 10 de marzo]

Sevillano, M. L. (1990): Hacia una didáctica de y con los medios, en Medina, A. y Sevillano, M.L. (coords.) DidácticaAdaptación. UNED. Vol. 2, 51-89. Madrid. 
Blanca Montt y Andrea Villarrubia

NOVASUR, TELEVISIÓN EDUCATIVA DE CHILE

\title{
PALABRAS CLAVE
}

Televisión educativa, recurso pedagógico, material audiovisual educativo, capacitación de profesores, trabajo colaborativo, videoteca educativa.

\section{KEYWORDS}

Educational Television, Pedagogical Resource, Cable in Classroom, Teacher's capacitation.

\section{PERFIL ACADÉMICO DE LAS AUTORAS}

Blanca Montt Fabres. Socióloga, Máster en Comunicación y Educación de la Universitat Autònoma de Barcelona. bmontt@yahoo.es

Andrea Villarrubia Martínez. Periodista. Máster en Educación y Nuevas Tecnologías de la Información y de la Comunicación. Universidad de Alicante. avillaru@yahoo. es

Dirección Postal:

\author{
Blanca Montt Fabres \\ Moneda 1020, piso 4 \\ Santiago \\ Chile
}

Fecha de entrega: 19.10.06

Fecha de aceptación: 23.12.06 